EPJ Web of Conferences 66, 08008 (2014)

DOI: $10.1051 /$ epjconf/ 20146608008

(C) Owned by the authors, published by EDP Sciences, 2014

\title{
CANDLES
}

\section{Search for neutrino-less double beta decay of ${ }^{48} \mathrm{Ca}$}

S. Umehara ${ }^{1, a}$, T. Kishimoto ${ }^{1,2}$, M. Nomachi ${ }^{1}$, S. Ajimura ${ }^{1}$, N. Nakatani ${ }^{1}$, K. Matsuoka ${ }^{1}$, K. Ichimura ${ }^{1}$, M. Saka1 ${ }^{1}$, T. Ishikawa ${ }^{1}$, D. Tanaka ${ }^{1}$, M. Tanaka ${ }^{1}$, S. Yoshida ${ }^{2}$, K. Suzuki ${ }^{2}$, G. Ito ${ }^{2}$, H. Kakubata ${ }^{2}$, W. Wang ${ }^{2}$, J. Takemoto ${ }^{2}$, W. M. Chan ${ }^{2}$, M. Doihara ${ }^{2}$, Y. Tamagawa ${ }^{3}$, I. Ogawa ${ }^{3}$, T. Ueno $^{3}$, S. Maeda ${ }^{3}$, A. Yamamoto ${ }^{3}$, S. Tomita ${ }^{3}$, G. Fujita ${ }^{3}$, A. Kawamura ${ }^{3}$, T. Harada ${ }^{3}$, K. Fushimi ${ }^{4}$, R. Hazama ${ }^{5}, \mathrm{H}$. Ohsumi $^{6}$, and K. Okada ${ }^{7}$

${ }^{1}$ Research Center for Nuclear Physics (RCNP), Osaka University, Ibaraki, Osaka 567-0047, Japan
${ }^{2}$ Graduate School of Science, Osaka University, Toyonaka, Osaka 560-0043, Japan
${ }^{3}$ Graduate School of Engineering, University of Fukui, Fukui 910-8507, Japan
${ }^{4}$ Faculty of Integrated Arts and Science, The University of Tokushima, Tokushima 770-8502, Japan
${ }^{5}$ Faculty of Human Environment, Osaka Sangyo University, Daito, Osaka 574-8530, Japan
${ }^{6}$ Faculty of Culture and Education, Saga University, Saga 840-8502, Japan
${ }^{7}$ Department of Computer Science and Engineering, Kyoto Sangyo University, Kyoto 603-8555, Japan

\begin{abstract}
CANDLES is the project to search for neutrino-less double beta decay $(0 v \beta \beta)$ of ${ }^{48} \mathrm{Ca}$. The observation of $0 v \beta \beta$ will prove existence of a massive Majorana neutrino. For the $0 v \beta \beta$ measurement, we need a low background condition because of a low decay rate of $0 v \beta \beta$. Now we installed the CANDLES III system at the Kamioka underground laboratory. The CANDLES III system realizes the low background condition by a characteristic structure and data analyses for background rejection. Here we report performances of the CANDLES III system.
\end{abstract}

\section{Double beta decay of ${ }^{48} \mathrm{Ca}$}

The neutrino-less double beta decay is acquiring great interest after the confirmation of neutrino oscillation which demonstrated nonzero neutrino mass. Measurement of $0 v \beta \beta$ provides a test for the Majorana nature of neutrinos and gives an absolute scale of the effective neutrino mass. Many experiments have been carried out so far and many projects have been proposed.

Among double beta decay nuclei, ${ }^{48} \mathrm{Ca}$ has an advantage of the highest $\mathrm{Q}_{\beta \beta}$-value (4.27 MeV). This large $\mathrm{Q}_{\beta \beta}$-value gives a large phase-space factor to enhance the $0 v \beta \beta$ rate and the least contribution from natural background radiations in the energy region of the $\mathrm{Q}_{\beta \beta}$-value. Therefore good signal to background ratio is ensured in the measurement of $0 v \beta \beta$. For measurement with sensitivity to the mass region indicated by neutrino oscillation measurements we have to prepare several tons of calcium. Then we proposed CANDLES(CAlcium fluoride for the study of Neutrinos and Dark matters by Low Energy Spectrometer) system[1].

\footnotetext{
ae-mail: umehara@rcnp.osaka-u.ac.jp
} 
a)Schematic view of CANDLES III(U.G.) with Light-Concentration System

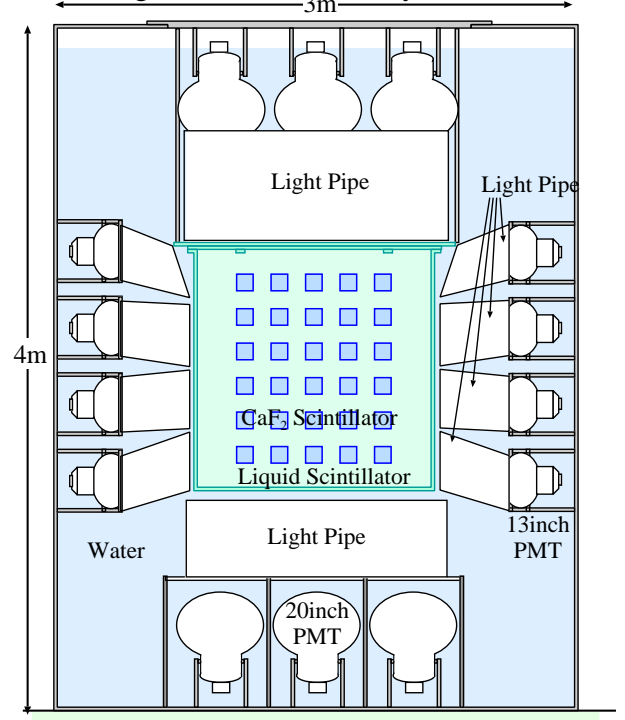

b)Energy Spectra

with/without Light-Concentration System

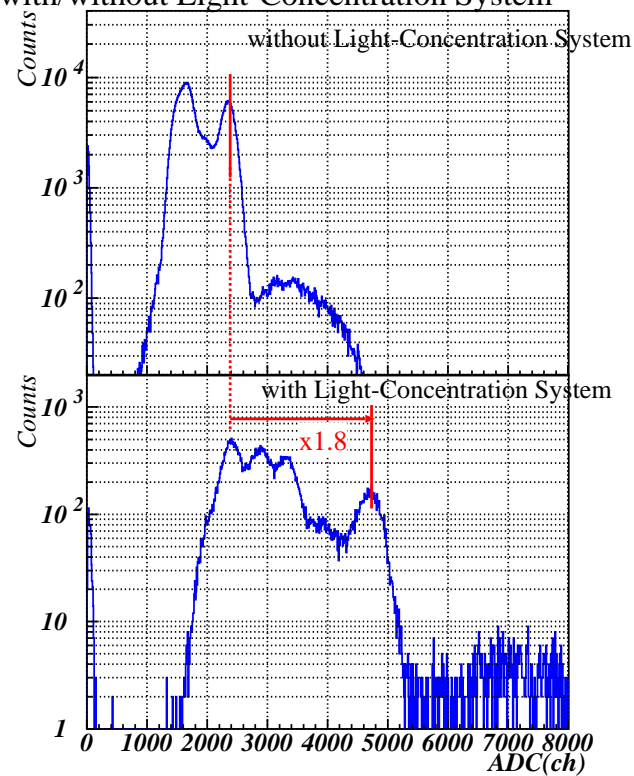

Figure 1. a)Schematic drawing of CANDLES III(U.G.). $\mathrm{CaF}_{2}$ (pure) scintillators are immersed in liquid scintillator. Scintillation lights from both $\mathrm{CaF}_{2}$ (pure) and liquid scintillator are viewed by large PMTs. b)The energy spectra of the reference $\mathrm{CaF}_{2}$ (pure) in the CANDLES III system. top) The energy spectrum without the lightconcentration system. The peak around $2500 \mathrm{ch}$ is due to $\alpha$-ray from radioactive contamination in the reference $\mathrm{CaF}_{2}$ (pure). bottom) The energy spectrum with the light-concentration system. The $\alpha$ peak is at $4700 \mathrm{ch}$ region. The light collection efficiency is 1.8 times larger than the one without the light-concentration system.

\section{CANDLES III at Kamioka observatory}

In the CANDLES system, $\mathrm{CaF}_{2}$ (pure) scintillators, which are main detectors, are immersed in liquid scintillator. The liquid scintillator acts as a $4 \pi$ active shield to veto backgrounds. Scintillation lights from $\mathrm{CaF}_{2}$ (pure) and liquid scintillators are viewed by large photomultiplier tubes. The $\mathrm{CaF}_{2}$ (pure) scintillator has a decay time of $1 \mu \mathrm{sec}$ although the liquid scintillator has a width of around a few tens nsec. Thus the signals from the $\mathrm{CaF}_{2}$ (pure) can be discriminated against the background signals on the liquid scintillator by observing pulse shapes.

We installed the detector system CANDLES III at Kamioka underground laboratory (2700 m.w.e.). Figure 1-a) shows a schematic view of the CANDLES III system. The CANDLES III system consists of $96 \mathrm{CaF}_{2}$ (pure) scintillators with total mass of $305 \mathrm{~kg}$ and liquid scintillator with total volume of $2 \mathrm{~m}^{3}$. The $96 \mathrm{CaF}_{2}$ (pure) scintillators are installed with 6 layers in a vessel for the liquid scintillator. Each layer has $16 \mathrm{CaF}_{2}$ (pure) scintillators. The $\mathrm{CaF}_{2}$ (pure) scintillators are suspended by wires from the ceiling of the liquid scintillator vessel. Scintillation lights from the $\mathrm{CaF}_{2}$ (pure) and liquid scintillator are viewed by 62 large photomultiplier tubes $(13$ " $\times 48$ and 20 " $\times 14)$. All detector modules are installed in a water tank of $3 \mathrm{~m}$ diameter and $4 \mathrm{~m}$ height[2]. 


\subsection{Background reduction}

As mentioned above, external backgrounds can be strongly limited because of the $4 \pi$ active shield by the liquid scintillator. The remaining backgrounds are following processes.

(a) two neutrino double beta decay $(2 v \beta \beta)$

(b) ${ }^{208} \mathrm{Tl} \longrightarrow{ }^{208} \mathrm{~Pb}$ (Th-chain)

(c) ${ }^{212} \mathrm{Bi} \underset{\beta}{\stackrel{\beta}{\longrightarrow}}{ }^{212} \mathrm{Po} \underset{\alpha}{\longrightarrow}{ }^{208} \mathrm{~Pb}$ (Th-chain)

The $2 v \beta \beta$ events (process (a)) can be rejected by a good energy resolution. The process (b) and process (c) can be rejected by a time correlation analysis and pulse shape analysis, respectively. Detail of the rejection of the process (c) are described in [3]. In this section we mention about study for the reduction of process (a) and (b).

\subsubsection{Light-concentration system}

In order to reject the $2 v \beta \beta$ events (process (a)), we need a good energy resolution. The good energy resolution will be realized by a light-concentration system and a wave-length shifter in the liquid scintillator. Details of the wave-length shifter are described in [4].

Recently we have installed the light-concentration system to improve the energy resolution. In the CANDLES III system, the photomultiplier tubes had small photo-coverage. In order to increase the photo-coverage, the light-concentration system was set between the photomultiplier tubes and the liquid scintillator vessel. The light-concentration system is shown in figure 1-a).

We checked the performance of the light-concentration system by using a reference $\mathrm{CaF}_{2}$ (pure), which has high radioactive contamination (Th-chain : $7 \mathrm{mBq} / \mathrm{kg}$ ). Figure 1-b) shows the energy spectra of the reference $\mathrm{CaF}_{2}$ (pure) with/without the light-concentration system. The position of the $\alpha$ peak rose from $2500 \mathrm{ch}$ region to $4700 \mathrm{ch}$ region. After correction of difference of 1 photo-electron gain between 2 spectra, we found that the light collection efficiency with the light-concentration system is 1.8 times larger than the one without the system. This corresponds to 0.9 p.e. $/ \mathrm{keV}$ in the number of photo-electron and satisfies a requirement for the CANDLES III system.

\subsection{2 ${ }^{208} \mathrm{Tl}$ rejection}

The other background candidate is the process (b) of ${ }^{208} \mathrm{Tl}$ events. ${ }^{208} \mathrm{Tl}$ has large $\mathrm{Q}_{\beta}$-value though it emits $2.6 \mathrm{MeV} \gamma$-ray. The probability that the high energy $\gamma$-rays are contained in a single $\mathrm{CaF}_{2}$ (pure) scintillator is small. However, the $0 v \beta \beta$ decay is extremely the rare process, thus the background has to be seriously considered.

In order to reject the ${ }^{208} \mathrm{Tl}$ events, we applied a time correlation analysis. The ${ }^{208} \mathrm{Tl}$ events has a preceding $\alpha$-decay with a half life of 3 minutes. Thus we can reject the ${ }^{208} \mathrm{Tl}$ events by identifying the preceding $\alpha$-ray. For identifying the $\alpha$-ray, we need the good position resolution and the pulse shape discrimination between $\alpha$ - and $\gamma$-rays.

The position resolution was tested by the CANDLES III system with the light-concentration system. The signal positions in the $\mathrm{CaF}_{2}$ (pure) scintillators were reconstructed by using pulse height information of the 62 photomultiplier tubes. Figure 2-a) shows the result of the position reconstruction. We can find that the events distribute at the positions of each $\mathrm{CaF}_{2}$ module. The peaks by the reconstructed position have $\sim 6 \sigma$ separation between the peaks by the next $\mathrm{CaF}_{2}$. The separation corresponds to a few centimeter for the position resolution. The separation satisfies a requirement for rejection of the ${ }^{208} \mathrm{Tl}$ event by identifying the preceding $\alpha$-ray. On the other hand, the pulse shape discrimination between $\alpha$ - and $\gamma$-rays is realized by pulse shape analysis. A clear discrimination is 

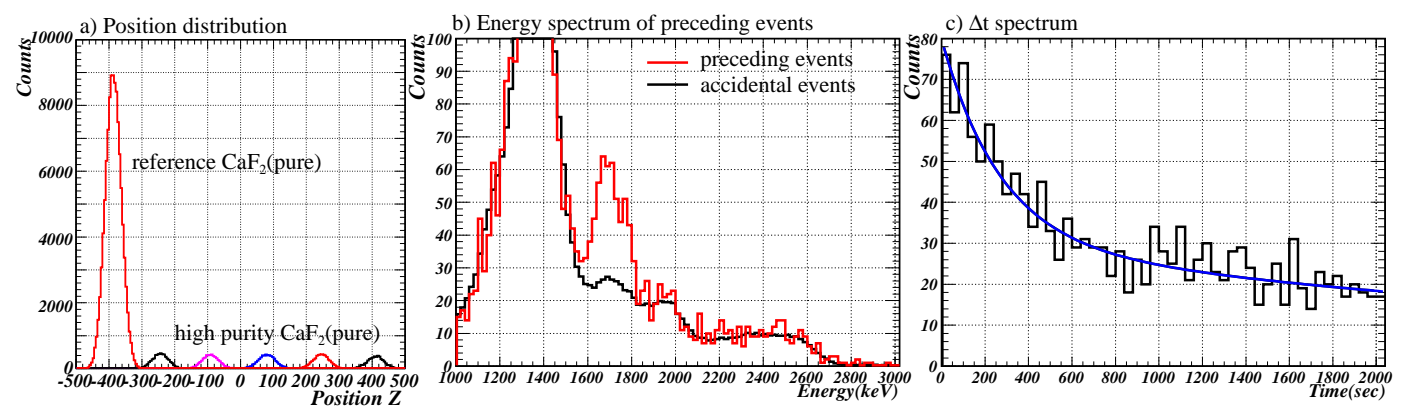

Figure 2. a) Result of the position reconstruction analysis on Z-axis. In the CANDLES III system, 6 layers of the $\mathrm{CaF}_{2}$ (pure) scintillators are installed on the $\mathrm{Z}$-axis. The left peak is corresponding to the reference $\mathrm{CaF}_{2}$ (pure). The peaks of the reconstructed position have $\sim 6 \sigma$ separation for each $\mathrm{CaF}_{2}$ (pure) scintillator. b) The energy spectra of the preceding events of ${ }^{208} \mathrm{Tl}$. Red (black) line corresponds to the preceding (accidental) events. The peak at $1.7 \mathrm{MeV}$ was due to ${ }^{212} \mathrm{Bi}$ decay $\left(\mathrm{E}_{\alpha}=6.1 \mathrm{MeV}\right)$. c) $\Delta t$ distribution between the preceding and delayed events. By fitting with two exponential function, we obtained the half-life of $187 \pm 56 \mathrm{sec}$.

obtained between $\alpha$ - and $\gamma$-rays. Rejection efficiency is $98 \%$ of ( $\gamma$-ray + liquid scintillator) events with $90 \%$ of acceptance for $\alpha$-ray.

Based on techniques of the position reconstruction and the pulse shape discrimination, we applied the time correlation analysis for ${ }^{208} \mathrm{Tl}$. The energy spectrum of the candidate events of the preceding $\alpha$ decay is shown in figure 2-b). The peak at $1.7 \mathrm{MeV}$ was likely due to $\alpha$-rays coming from the preceding $\alpha$ decay $\left({ }^{212} \mathrm{Bi}: E_{\alpha}=6.1 \mathrm{MeV}\right)$. To confirm the origin of the peak, we analyzed the distribution of time lag $\Delta t$ between the preceding and the delayed events. The time lag $\Delta t$ distribution of the preceding events with energy of $1.6-1.8 \mathrm{MeV}$ is shown as figure 2-c). In order to obtain the half-life, we fitted the time spectrum with two exponential function. The half-life derived from the $\Delta t$ distribution was 187 $\pm 56 \mathrm{sec}$. The calculated half-life nearly agreed with one of ${ }^{208} \mathrm{Tl}(183 \mathrm{sec})$. Thus it was concluded that the peak at $1.7 \mathrm{MeV}$ was due to ${ }^{212} \mathrm{Bi} \alpha$-rays and we found that ${ }^{208} \mathrm{Tl}$ can be rejected by the time correlation analysis.

\section{Conclusion}

We have developed CANDLES in order to explore the mass region of $\sim 30 \mathrm{meV}$. Now the CANDLES III system was installed at the Kamioka underground laboratory. By improvement of the detector system and the pulse shape analyses, we can reduce the background events from $2 v \beta \beta$ and ${ }^{208} \mathrm{Tl}$. By estimating the results for the background reductions, the system will achieve the background free measurement. The expected sensitivity of the CANDLES III system is $0.5 \mathrm{eV}$ for effective neutrino mass. In near future, CANDLES will be scaled up for a high sensitive measurement. This system has no problem to construct the large system.

\section{References}

[1] T. Kishimoto et al., Candles for the study of beta beta decay of Ca-48, in Proc. of 4th Workshop on Neutrino Oscillations and their Origin (2003), p. 338

[2] S. Umehara et al., AIP Conf. Proc. 1235, 287 (2010)

[3] S. Umehara et al., Phys. Rev. C 78, 058501 (2008)

[4] S. Yoshida et al., Nucl. Instrum. Meth. A601, 282 (2009) 